\title{
Conformal Form of Pseudo-Riemannian Metrics by Normal Coordinate Transformations II
}

\author{
A. C. V. V. de Siqueira * \\ Departamento de Educação \\ Universidade Federal Rural de Pernambuco \\ 52.171-900, Recife, PE, Brazil.
}

\begin{abstract}
In this paper, we have reintroduced a new approach to conformal geometry developed and presented in two previous papers, in which we show that all n-dimensional pseudo-Riemannian metrics are conformal to a flat n-dimensional manifold as well as an n-dimensional manifold of constant curvature when Riemannian normal coordinates are well-behaved in the origin and in their neighborhood. This was based on an approach developed by French mathematician Elie Cartan. As a consequence of geometry, we have reintroduced the classical and quantum angular momenta of a particle and present new interpretations. We also show that all n-dimensional pseudo-Riemannian metrics can be embedded in a hyper-cone of a flat n+2-dimensional manifold.
\end{abstract}

*E-mail: acvvs@ded.ufrpe.br 


\section{Introduction}

In this paper, we reintroduce a new approach to conformal geometry developed and presented in two previous papers, [1], [2]. This was based on an approach developed by French mathematician Elie Cartan, [3], [4], [5]. Some classical and quantum results are reintroduced from a new viewpoint.

This paper is organized as follows. In Sec. 2, we present normal coordinates and elements of differential geometry. In Sec. 3, we show that, in normal coordinates, all n-dimensional pseudo-Riemannian metrics that are well-behaved in origin and in their neighborhood are conformal to a flat ndimensional manifold and an n-dimensional manifold of constant curvature. In Sec. 4, this result is used in the Cartan solution for a space of constant curvature. In Sec. 5, we present more differential geometry, introducing normal tensors to build the Cartan solution for a general pseudo-Riemannian metric. In Sec. 6, we make an embedding of all n-dimensional pseudo-Riemannian manifolds of constant curvature in a flat $n+1$-dimensional manifold, obtaining the quantum angular momentum operator of a particle as a consequence of geometry. In Sec. 7, a new geometric postulate is announced and some new classical physical principles are developed. In Sec. 8, we make an embedding of all n-dimensional pseudo-Riemannian metrics that obey previously presented conditions into a hyper-cone of a flat $n+2$-dimensional space.

\section{Normal Coordinates}

In this section, we briefly present normal coordinates and review some elements of differential geometry for an n-dimensional pseudo-Riemannian manifold, [3], [4], [5].

Let us consider the line element

$$
d s^{2}=G_{\Lambda \Pi} d u^{\Lambda} d u^{\Pi}
$$

with

$$
G_{\Lambda \Pi}=E_{\Lambda}^{(\mathbf{A})} E_{\Pi}^{(\mathbf{B})} \eta_{(\mathbf{A})(\mathbf{B})}
$$

in which $\eta_{(\mathbf{A})(\mathbf{B})}$ and $E_{\Lambda}^{(\mathbf{A})}$ are flat metric and vielbein components, respectively.

We choose each $\eta_{(\mathbf{A})(\mathbf{B})}$ as plus or minus Kronecker's delta function. Let us 
give the 1-form $\omega^{(\mathbf{A})}$ by

$$
\omega^{(\mathbf{A})}=d u^{\Lambda} E_{\Lambda}^{(\mathbf{A})} .
$$

We now define Riemannian normal coordinates by

$$
u^{\Lambda}=v^{\Lambda} t
$$

then

$$
d u^{\Lambda}=v^{\Lambda} d t+t d v^{\Lambda}
$$

Placing (2.5) in (2.3)

$$
\omega^{(\mathbf{A})}=t d v^{\Lambda} E_{\Lambda}^{(\mathbf{A})}+d t v^{\Lambda} E_{\Lambda}^{(\mathbf{A})} .
$$

Let us define

$$
z^{(\mathbf{A})}=v^{\Lambda} E_{\Lambda}^{(\mathbf{A})}
$$

so that

$$
\omega^{(\mathbf{A})}=d t z^{(\mathbf{A})}+t d z^{(\mathbf{A})}+t E^{\Pi(\mathbf{A})} \frac{\partial E_{\Pi(\mathbf{B})}}{\partial z^{(\mathbf{C})}} z^{(\mathbf{B})} d z^{(\mathbf{C})} .
$$

We now make

$$
A^{(\mathbf{A})_{(\mathbf{B})(\mathbf{C})}}=t E^{\Pi(\mathbf{A})} \frac{\partial E_{\Pi(\mathbf{B})}}{\partial z^{(\mathbf{C})}},
$$

then

$$
\varpi^{(\mathbf{A})}=t d z^{(\mathbf{A})}+A^{(A)_{(\mathbf{B})(\mathbf{C})}} z^{(\mathbf{B})} d z^{(\mathbf{C})}
$$

with

$$
\omega^{(\mathbf{A})}=d t z^{(\mathbf{A})}+\varpi^{(\mathbf{A})} .
$$

At $t=0$, we have

$$
\begin{gathered}
A^{(A)_{(\mathbf{B})(\mathbf{C})}}\left(t=0, z^{(\mathbf{D})}\right)=0, \\
\varpi^{(\mathbf{A})}\left(t=0, z^{(\mathbf{D})}\right)=0,
\end{gathered}
$$

and

$$
\omega^{(\mathbf{A})}\left(t=0, z^{(\mathbf{D})}\right)=d t z^{(\mathbf{A})} .
$$

We conclude that $\omega^{(\mathbf{A})}$ is the one-form associated to the normal coordinate $u^{\Lambda}, z^{(\mathbf{A})}$ is associated to the local coordinate $v^{\Lambda}$ of a local basis and $\varpi^{(\mathbf{A})}$ is the one-form associated to the one-form $d z^{(\mathbf{A})}$.

In an $\mathrm{n}+1$-manifold, consider a coordinate system given by $\left(t, z^{(\mathbf{A})}\right)$. For each value of $t$, we have a hyper-surface in which $d t=0$ on each. We are 
interested in the hyper-surface with $t=1$. On this hyper-surface, we verify the following equality:

$$
\omega^{(\mathbf{A})}(t=1, z)=\varpi^{(\mathbf{A})}(t=1, z) .
$$

Consider the following expression on a vielbein basis:

$$
d \omega^{(\mathbf{A})}=-\omega_{(\mathbf{B})}^{(\mathbf{A})} \wedge \omega^{(\mathbf{B})} .
$$

The expression is invariant by coordinate transformations.

Now consider the map $\Phi$ between two manifolds $\mathrm{M}$ and $\mathrm{N}$, and consider two subsets, $\mathrm{U}$ of $\mathrm{M}$ and $\mathrm{V}$ of $\mathrm{N}$. Then,

$$
\Phi: U \longrightarrow V \text {. }
$$

We now define pull-back as follows: [4],

$$
\Phi^{*}: F^{p}(V) \longrightarrow F^{p}(U),
$$

so that $\Phi^{*}$ sends p-forms to other p-forms.

It is well known that the exterior derivative commutes with pull-back, so that

$$
\Phi^{*}\left(d \omega_{(\mathbf{B})}^{(\mathbf{A})}\right)=d \Phi^{*}\left(\omega_{(\mathbf{B})}^{(\mathbf{A})}\right)
$$

and

$$
\Phi^{*}\left(d \omega^{(\mathbf{A})}\right)=d \Phi^{*}\left(\omega^{(\mathbf{A})}\right)
$$

We also have

$$
\Phi^{*}\left(\omega_{(\mathbf{B})}^{(\mathbf{A})} \wedge \omega^{(\mathbf{B})}\right)=\Phi^{*}\left(\omega_{(\mathbf{B})}^{(\mathbf{A})}\right) \wedge \Phi^{*}\left(\omega^{(\mathbf{B})}\right) .
$$

The equation (2.11) can be seen as a pull-back,

$$
\Phi^{*}\left(\omega^{(\mathbf{A})}\right)=d t z^{(\mathbf{A})}+\varpi^{(\mathbf{A})} .
$$

By a simple calculation, it can be shown that

$$
\Phi^{*}\left(\omega_{(\mathbf{B})}^{(\mathbf{A})}\right)=\varpi_{(\mathbf{B})}^{(\mathbf{A})} .
$$

Note that $d t=0$ for $\varpi^{(\mathbf{A})}$ and $\varpi_{(\mathbf{B})}^{(\mathbf{A})}$. 
By the exterior derivative of (2.22), we obtain

$$
\begin{aligned}
d\left(\Phi^{*}\left(\omega^{(\mathbf{A})}\right)\right)=d\left(d t z^{(\mathbf{A})}+\varpi^{(\mathbf{A})}\right) & =d z^{(\mathbf{A})} \wedge(d t) \\
& +d t \wedge \frac{\partial\left(\varpi^{(\mathbf{A})}\right)}{\partial(t)}
\end{aligned}
$$

+ terms not involving $d t$.

Making a pull-back of (2.16) and using (2.21), we have

$$
\Phi^{*}\left(d \omega^{(\mathbf{A})}\right)=\Phi^{*}\left(-\omega_{(\mathbf{B})}^{(\mathbf{A})} \wedge \omega^{(\mathbf{B})}\right)=-\Phi^{*}\left(\omega_{(\mathbf{B})}^{(\mathbf{A})}\right) \wedge \Phi^{*}\left(\omega^{(\mathbf{B})}\right) .
$$

Using (2.20), (2.23), (2.24) and (2.25), we obtain

$$
\frac{\partial \varpi^{(\mathbf{A})}}{\partial t}=d z^{(\mathbf{A})}+\varpi_{(\mathbf{B})}^{(\mathbf{A})} z^{(\mathbf{D})} .
$$

By a similar procedure to (2.19) and using the Cartan second structure equation, we obtain the following result:

$$
\frac{\partial \varpi_{(\mathbf{A})(\mathbf{B})}}{\partial t}=R_{(\mathbf{A})(\mathbf{B})(\mathbf{C})(\mathbf{D})} z^{(\mathbf{C})} \varpi^{(\mathbf{A})} .
$$

Making a new partial derivative of (2.26), two partial derivatives of (2.10), comparing the results and using (2.27), we have the following equation:

$$
\frac{\partial^{2} A_{(\mathbf{A})(\mathbf{C})(\mathbf{D})}}{\partial t^{2}}=t z^{(\mathbf{B})} R_{(\mathbf{A})(\mathbf{B})(\mathbf{C})(\mathbf{D})}+z^{(\mathbf{L})} z^{(\mathbf{M})} R_{(\mathbf{A})(\mathbf{L})(\mathbf{M})(\mathbf{N})} A_{(\mathbf{P})(\mathbf{C})(\mathbf{D})} \eta^{(\mathbf{N})(\mathbf{P})} .
$$

Rewriting (2.28) with indices (C) and (D) permuted, we obtain the result

$$
\frac{\partial^{2} A_{(\mathbf{A})(\mathbf{D})(\mathbf{C})}}{\partial t^{2}}=t z^{(\mathbf{B})} R_{(\mathbf{A})(\mathbf{B})(\mathbf{D})(\mathbf{C})}+z^{(\mathbf{L})} z^{(\mathbf{M})} R_{(\mathbf{A})(\mathbf{L})(\mathbf{M})(\mathbf{N})} A_{(\mathbf{P})(\mathbf{D})(\mathbf{C})} \eta^{(\mathbf{N})(\mathbf{P})} .
$$

Adding (2.28) and (2.29) and using the curvature symmetries, we have

$$
A_{(\mathbf{A})(\mathbf{C})(\mathbf{D})}+A_{(\mathbf{A})(\mathbf{D})(\mathbf{C})}=0,
$$


which is true for all $t$.

Then,

$$
A_{(\mathbf{A})(\mathbf{C})(\mathbf{D})}=-A_{(\mathbf{A})(\mathbf{D})(\mathbf{C})},
$$

such that we can rewrite $(2.10)$ as

$$
\varpi^{(\mathbf{A})}=t d z^{(\mathbf{A})}+\frac{1}{2} A^{(A)_{(\mathbf{B})(\mathbf{C})}}\left(z^{(\mathbf{B})} d z^{(\mathbf{C})}-z^{(\mathbf{C})} d z^{(\mathbf{B})}\right) .
$$

Let us define

$$
A_{(\mathbf{A})(\mathbf{C})(\mathbf{D})}=z^{(\mathbf{B})} B_{(\mathbf{A})(\mathbf{B})(\mathbf{C})(\mathbf{D})} .
$$

The following result is obtained by placing (2.33) in (2.28):

$\frac{\partial^{2} B_{(\mathbf{A})(\mathbf{B})(\mathbf{C})(\mathbf{D})}}{\partial t^{2}}=t R_{(\mathbf{A})(\mathbf{B})(\mathbf{C})(\mathbf{D})}+z^{(\mathbf{L})} z^{(\mathbf{M})} R_{(\mathbf{A})(\mathbf{B})(\mathbf{L})(\mathbf{N})} B_{(\mathbf{P})(\mathbf{M})(\mathbf{C})(\mathbf{D})} \eta^{(\mathbf{N})(\mathbf{P})}$.

We now rewrite $(2.34)$ as

$\frac{\partial^{2} B_{(\mathbf{B})(\mathbf{A})(\mathbf{C})(\mathbf{D})}}{\partial t^{2}}=t R_{(\mathbf{B})(\mathbf{A})(\mathbf{C})(\mathbf{D})}+z^{(\mathbf{L})} z^{(\mathbf{M})} R_{(\mathbf{B})(\mathbf{A})(\mathbf{L})(\mathbf{N})} B_{(\mathbf{P})(\mathbf{M})(\mathbf{C})(\mathbf{D})} \eta^{(\mathbf{N})(\mathbf{P})}$.

Adding (2.34) and (2.35) and using the curvature symmetries, we obtain the solution

$$
B_{(\mathbf{A})(\mathbf{B})(\mathbf{C})(\mathbf{D})}+B_{(\mathbf{B})(\mathbf{A})(\mathbf{C})(\mathbf{D})}=\text { const. }
$$

for all $t$.

We use (2.12) and (2.33) in (2.36) to obtain

$$
B_{(\mathbf{A})(\mathbf{B})(\mathbf{C})(\mathbf{D})}+B_{(\mathbf{B})(\mathbf{A})(\mathbf{C})(\mathbf{D})}=0 .
$$

For future use, we present the line element on the hyper-surface

$$
d s^{2}=\eta_{(\mathbf{A})(\mathbf{B})} \varpi^{(\mathbf{A})} \varpi^{(\mathbf{B})} .
$$

From $(2.31),(2.33)$ and $(2.37)$, we conclude that $B_{(\mathbf{A})(\mathbf{B})(\mathbf{C})(\mathbf{D})}$ has the same symmetries as the Riemann curvature tensor

$$
B_{(\mathbf{A})(\mathbf{B})(\mathbf{C})(\mathbf{D})}=-B_{(\mathbf{B})(\mathbf{A})(\mathbf{C})(\mathbf{D})}=-B_{(\mathbf{A})(\mathbf{B})(\mathbf{D})(\mathbf{C})} .
$$


Using (2.31) and (2.33), we have

$$
\begin{array}{r}
A_{(\mathbf{A})(\mathbf{C})(\mathbf{D})} d z^{(\mathbf{A})} z^{(\mathbf{C})} d z^{(\mathbf{D})}= \\
+\frac{1}{4} B_{(\mathbf{A})(\mathbf{B})(\mathbf{C})(\mathbf{D}) .} \\
.\left(z^{(\mathbf{B})} d z^{(\mathbf{A})}-z^{(\mathbf{A})} d z^{(\mathbf{B})}\right) . \\
.\left(z^{(\mathbf{C})} d z^{(\mathbf{D})}-z^{(\mathbf{D})} d z^{(\mathbf{C})}\right) .
\end{array}
$$

We now construct the line element of the hyper-surface. With direct use of (2.32) and (2.40)in (2.38), we have

$$
\begin{array}{r}
d s^{2}=t^{2} \eta_{(\mathbf{A})(\mathbf{B})} d z^{(\mathbf{A})} d z^{(\mathbf{B})}+ \\
+\frac{1}{2}\left\{\frac{1}{2} t \epsilon_{(\mathbf{B})} B_{(\mathbf{A})(\mathbf{B})(\mathbf{C})(\mathbf{D})}+\right. \\
\left.+\eta^{(\mathbf{M})(\mathbf{N})} A_{(\mathbf{M})(\mathbf{B})(\mathbf{A})} A_{(\mathbf{N})(\mathbf{C})(\mathbf{D})}\right\} . \\
.\left(z^{(\mathbf{B})} d z^{(\mathbf{A})}-z^{(\mathbf{A})} d z^{(\mathbf{B})}\right)\left(z^{(\mathbf{C})} d z^{(\mathbf{D})}-z^{(\mathbf{D})} d z^{(\mathbf{C})}\right) .
\end{array}
$$

The line element of the manifold and the hyper-surface are equal at $t=1$, where $u^{\Lambda}=v^{\Lambda}$,

$$
d s^{2}=d s^{2}
$$

and

$$
\begin{array}{r}
d s^{2}=\eta_{(\mathbf{A})(\mathbf{B})} d z^{(\mathbf{A})} d z^{(\mathbf{B})}+ \\
+\frac{1}{2}\left\{\frac{1}{2} \epsilon_{(\mathbf{B})} B_{(\mathbf{A})(\mathbf{B})(\mathbf{C})(\mathbf{D})}+\right. \\
\left.+\eta^{(\mathbf{M})(\mathbf{N})} A_{(\mathbf{M})(\mathbf{B})(\mathbf{A})} A_{(\mathbf{N})(\mathbf{C})(\mathbf{D})}\right\} . \\
.\left(z^{(\mathbf{B})} d z^{(\mathbf{A})}-z^{(\mathbf{A})} d z^{(\mathbf{B})}\right)\left(z^{(\mathbf{C})} d z^{(\mathbf{D})}-z^{(\mathbf{D})} d z^{(\mathbf{C})}\right) .
\end{array}
$$

Note that (2.43) is not an approximation of (2.1); the equations are equal. 


\section{Conformal Form of Riemannian Metrics}

Sometimes, it is possible to write the metric (2.43) in the particular form (3.1), as follows:

$$
\begin{array}{r}
d s^{2}=\eta_{(\mathbf{a})(\mathbf{b})} d z^{(\mathbf{a})} d z^{(\mathbf{b})}+ \\
+\left\{\eta_{(\mathbf{0})(\mathbf{0})}+\frac{1}{2}\left[\frac{1}{2} \epsilon_{(\mathbf{B})} B_{(\mathbf{A})(\mathbf{B})(\mathbf{C})(\mathbf{D})}+\right.\right. \\
\left.+\eta^{(\mathbf{M})(\mathbf{N})} A_{(\mathbf{M})(\mathbf{B})(\mathbf{A})} A_{(\mathbf{N})(\mathbf{C})(\mathbf{D})}\right] . \\
\left..\left(z^{(\mathbf{B})} \frac{d z^{(\mathbf{A})}}{d \tau}-z^{(\mathbf{A})} \frac{d z^{(\mathbf{B})}}{d \tau}\right)\left(z^{(\mathbf{C})} \frac{d z^{(\mathbf{D})}}{d \tau}-z^{(\mathbf{D})} \frac{d z^{(\mathbf{C})}}{d \tau}\right)\right\} d \tau^{2},
\end{array}
$$

in which $(a),(b) \neq 0$.

Defining

$$
\begin{aligned}
& d \rho^{2}=\left\{\eta_{(\mathbf{0})(\mathbf{0})}+\frac{1}{2}\left[\frac{1}{2} \epsilon_{(\mathbf{B})} B_{(\mathbf{A})(\mathbf{B})(\mathbf{C})(\mathbf{D})}+\right.\right. \\
& \left.+\eta^{(\mathbf{M})(\mathbf{N})} A_{(\mathbf{M})(\mathbf{B})(\mathbf{A})} A_{(\mathbf{N})(\mathbf{C})(\mathbf{D})}\right] . \\
& \left..\left(z^{(\mathbf{B})} \frac{d z^{(\mathbf{A})}}{d \tau}-z^{(\mathbf{A})} \frac{d z^{(\mathbf{B})}}{d \tau}\right)\left(z^{(\mathbf{C})} \frac{d z^{(\mathbf{D})}}{d \tau}-z^{(\mathbf{D})} \frac{d z^{(\mathbf{C})}}{d \tau}\right)\right\} d \tau^{2},
\end{aligned}
$$

then, (3.1) can be rewritten as

$$
d s^{2}=d \rho^{2}+\eta_{(\mathbf{a})(\mathbf{b})} d z^{(\mathbf{a})} d z^{(\mathbf{b})} .
$$

We now write (2.43) as

$$
\begin{array}{r}
d s^{2}=\eta_{(\mathbf{A})(\mathbf{B})} d z^{(\mathbf{A})} d z^{(\mathbf{B})}+ \\
+\left\{\frac { 1 } { 2 } \left[\frac{1}{2} \epsilon_{(\mathbf{B})} B_{(\mathbf{A})(\mathbf{B})(\mathbf{C})(\mathbf{D})}+\right.\right. \\
\left.\left.+\eta^{(\mathbf{M})(\mathbf{N})} A_{(\mathbf{M})(\mathbf{B})(\mathbf{A})} A_{(\mathbf{N})(\mathbf{C})(\mathbf{D})}\right]\right\} . \\
.\left(z^{(\mathbf{B})} \frac{d z^{(\mathbf{A})}}{d s}-z^{(\mathbf{A})} \frac{d z^{(\mathbf{B})}}{d s}\right)\left(z^{(\mathbf{C})} \frac{d z^{(\mathbf{D})}}{d s}-z^{(\mathbf{D})} \frac{d z^{(\mathbf{C})}}{d s}\right) d s^{2} .
\end{array}
$$


This can be written in the following form:

$$
\begin{aligned}
& {\left[1-\frac{1}{2}\left[\frac{1}{2} \epsilon_{(\mathbf{B})} B_{(\mathbf{A})(\mathbf{B})(\mathbf{C})(\mathbf{D})}+\right.\right.} \\
& \left.+\eta^{(\mathbf{M})(\mathbf{N})} A_{(\mathbf{M})(\mathbf{B})(\mathbf{A})} A_{(\mathbf{N})(\mathbf{C})(\mathbf{D})}\right] . \\
& \left..\left(z^{(\mathbf{B})} \frac{d z^{(\mathbf{A})}}{d s}-z^{(\mathbf{A})} \frac{d z^{(\mathbf{B})}}{d s}\right)\left(z^{(\mathbf{C})} \frac{d z^{(\mathbf{D})}}{d s}-z^{(\mathbf{D})} \frac{d z^{(\mathbf{C})}}{d s}\right)\right] d s^{2} \\
& =\eta_{(\mathbf{A})(\mathbf{B})} d z^{(\mathbf{A})} d z^{(\mathbf{B})} \text {. }
\end{aligned}
$$

We now define the function

$$
L^{(\mathbf{B})(\mathbf{A})}=\left(z^{(\mathbf{B})} \frac{d z^{(\mathbf{A})}}{d s}-z^{(\mathbf{A})} \frac{d z^{(\mathbf{B})}}{d s}\right)
$$

which is the classical angular momentum of a free particle.

The line element (3.6) can assume the form

$$
\begin{array}{r}
\left\{1+\frac{1}{2}\left[\frac { 1 } { 2 } \left(\epsilon_{(\mathbf{B})} B_{(\mathbf{A})(\mathbf{B})(\mathbf{C})(\mathbf{D})}+\right.\right.\right. \\
+\eta^{(\mathbf{M})(\mathbf{N})} A_{(\mathbf{M})(\mathbf{B})(\mathbf{A})} A_{(\mathbf{N})(\mathbf{C})(\mathbf{D})]} \\
\left..\left(L^{\mathbf{A})(\mathbf{B})} L^{\mathbf{C})(\mathbf{D})}\right)\right\} d s^{2} \\
=\left(\eta_{(\mathbf{A})(\mathbf{B})} d z^{(\mathbf{A})} d z^{(\mathbf{B})} .\right.
\end{array}
$$

We now define the function

$$
\begin{array}{r}
\exp (-2 \sigma)=\left\{1+\frac{1}{2}\left[\frac { 1 } { 2 } \left(\epsilon_{(\mathbf{B})} B_{(\mathbf{A})(\mathbf{B})(\mathbf{C})(\mathbf{D})}\right.\right.\right. \\
\left.\left.+\eta^{(\mathbf{M})(\mathbf{N})} A_{(\mathbf{M})(\mathbf{B})(\mathbf{A})} A_{(\mathbf{N}))(\mathbf{C})(\mathbf{D})}\right)\right] . \\
\left.. L^{(\mathbf{A})(\mathbf{B})} L^{(\mathbf{C})(\mathbf{D})}\right\}
\end{array}
$$

then, the line element assumes the form

$$
d s^{2}=\exp (2 \sigma) \eta_{(\mathbf{A})(\mathbf{B})} d z^{(\mathbf{A})} d z^{(\mathbf{B})} .
$$


When transformations such as (3.2) are possible, (3.4) is a flat metric with the time changed and is equivalent to the original metric. In other words, (3.4) is the original line element of the curved manifold written in a flat form.

The metric (3.10) is conformal to a flat manifold as well as a manifold of constant curvature when the normal coordinate transformations are wellbehaved in the origin and in their neighborhood. A Riemannian normal transformation and its inverse are well-behaved in the region in which geodesics are not mixed. Points in which geodesics approach each other or mix are known as conjugate points of Jacobi's fields. Jacobi's fields can be used for this purpose. Although this is an important problem, we do not make further considerations about the regions in which (3.4) and (3.10) are valid.

In the next section, we present the Cartan solution for the case in which curvature is constant. For the Cartan solution to a general metric, more geometric objects are necessary, such as normal tensors. This will be presented in section 5 .

\section{Cartan's Solution for Constant Curvature}

In this section, we present the Cartan solution for constant curvature. The calculation is very simple and was done in [3], and reproduced in detail in [4]. Our objective in this section is only to place the Cartan solution in the forms (3.4) and (3.10).

Cartan used the signature $(+,+,+\ldots .+)$ and obtained the line element

$$
\begin{array}{r}
d s^{2}=\sum_{k=1}^{n}\left(\varpi^{\mathbf{k}}\right)^{2}=\sum_{k=1}^{n}\left(d v^{\mathbf{k}}\right)^{2}+ \\
-\left[\frac{|K| \mathbf{r}^{\mathbf{2}}-\mathbf{S}^{\mathbf{2}}(\mathbf{r} \sqrt{|\mathbf{K}|} \mathbf{t})}{|K| \mathbf{r}^{\mathbf{4}}}\right] \sum_{i<j}\left(v^{\mathbf{i}} d v^{\mathbf{j}}-v^{\mathbf{j}} d v^{\mathbf{i}}\right)^{2}
\end{array}
$$

in which when $K>0$

$$
\mathbf{S}=\sin (\sqrt{|\mathbf{K}|} \mathbf{t})
$$

and when $K<0$

$$
\mathbf{S}=\sinh (\sqrt{|\mathbf{K}|} \mathbf{t})
$$


We write (4.1) in the form (3.1)

$$
\begin{array}{r}
d s^{2}=\sum_{k=1}^{n}\left(\varpi^{\mathbf{k}}\right)^{2}=\sum_{k=1}^{n}\left(d v^{\mathbf{k}}\right)^{2} \\
-\left[\frac{|K| \mathbf{r}^{\mathbf{2}}-\mathbf{S}^{\mathbf{2}}(\mathbf{r} \sqrt{|\mathbf{K}|} \mathbf{t})}{|K| \mathbf{r}^{\mathbf{4}}}\right] \sum_{i<j}\left(v^{\mathbf{i}} \frac{d v^{\mathbf{j}}}{d \tau}-v^{\mathbf{j}} \frac{d v^{\mathbf{i}}}{d \tau}\right)^{2} d \tau^{2} .
\end{array}
$$

Consider the following function:

$$
l^{\mathbf{i} \mathbf{j}}=\sum_{i<j}\left(v^{\mathbf{i}} \frac{d v^{\mathbf{j}}}{d \tau}-v^{\mathbf{j}} \frac{d v^{\mathbf{i}}}{d \tau}\right)^{2} .
$$

Using (4.5) in (4.4), we have

$$
\begin{array}{r}
d s^{2}=\sum_{k=1}^{n}\left(\varpi^{\mathbf{k}}\right)^{2}=\sum_{k=1}^{n}\left(d v^{\mathbf{k}}\right)^{2} \\
-\left[\frac{|K| \mathbf{r}^{\mathbf{2}}-\mathbf{S}^{\mathbf{2}}(\mathbf{r} \sqrt{|\mathbf{K}|} \mathbf{t})}{|K| \mathbf{r}^{4}}\right] \sum_{i<j}\left(l^{\mathbf{i j}}\right)^{2} d \tau^{2} .
\end{array}
$$

Sometimes, we can put $d v^{1}=d \tau$. In this case, (4.6) can be written in the form

$$
\begin{array}{r}
d s^{2}=\sum_{k=1}^{n}\left(\varpi^{\mathbf{k}}\right)^{2}=\sum_{k=2}^{n}\left(d v^{\mathbf{k}}\right)^{2} \\
+\left\{1-\left[\frac{|K| \mathbf{r}^{\mathbf{2}}-\mathbf{S}^{\mathbf{2}}(\mathbf{r} \sqrt{|\mathbf{K}|} \mathbf{t})}{|K| \mathbf{r}^{\mathbf{4}}}\right] \sum_{i<j}\left(l^{\mathbf{i j}}\right)^{2}\right\} d \tau^{2} .
\end{array}
$$

Defining

$$
d \rho^{2}=\left\{1-\left[\frac{|K| \mathbf{r}^{\mathbf{2}}-\mathbf{S}^{\mathbf{2}}(\mathbf{r} \sqrt{|\mathbf{K}|} \mathbf{t})}{|K| \mathbf{r}^{\mathbf{4}}}\right] \sum_{i<j}\left(l^{\mathbf{i j}}\right)^{2}\right\} d \tau^{2},
$$


and placing it in (4.6), we obtain

$$
d s^{2}=d \rho^{2}+\sum_{k=2}^{n}\left(d v^{\mathbf{k}}\right)^{2},
$$

in which (4.9) has the same form as (3.4).

We now write (4.1) in the form (3.10). For this, we change (4.1) as follows:

$$
\begin{array}{r}
d s^{2}=\sum_{k=1}^{n}\left(\varpi^{\mathbf{k}}\right)^{2}=\sum_{k=1}^{n}\left(d v^{\mathbf{k}}\right)^{2}+ \\
-\left[\frac{|K| \mathbf{r}^{\mathbf{2}}-\mathbf{S}^{\mathbf{2}}(\mathbf{r} \sqrt{|\mathbf{K}|} \mathbf{t})}{|K| \mathbf{r}^{\mathbf{4}}}\right] \sum_{i<j}\left(v^{\mathbf{i}} \frac{d v^{\mathbf{j}}}{d s}-v^{\mathbf{j}} \frac{d v^{\mathbf{i}}}{d s}\right)^{2} d s^{2} .
\end{array}
$$

We see that (4.10) has the form of (3.5).

Defining

$$
L^{(\mathbf{i})(\mathbf{j})}=\left(z^{(\mathbf{i})} \frac{d z^{(\mathbf{j})}}{d s}-z^{(\mathbf{j})} \frac{d z^{(\mathbf{i})}}{d s}\right) .
$$

and placing (4.11) in (4.10), we obtain

$$
\begin{array}{r}
d s^{2}=\sum_{k=1}^{n}\left(\varpi^{\mathbf{k}}\right)^{2}=\sum_{k=1}^{n}\left(d v^{\mathbf{k}}\right)^{2}+ \\
-\left[\frac{|K| \mathbf{r}^{\mathbf{2}}-\mathbf{S}^{\mathbf{2}}(\mathbf{r} \sqrt{|\mathbf{K}|} \mathbf{t})}{|K| \mathbf{r}^{\mathbf{4}}}\right] \sum_{i<j}\left(L^{(\mathbf{i})(\mathbf{j})}\right)^{2} d s^{2},
\end{array}
$$

which is equivalent to

$$
\left\{1+\left[\frac{|K| \mathbf{r}^{\mathbf{2}}-\mathbf{S}^{\mathbf{2}}(\mathbf{r} \sqrt{|\mathbf{K}|} \mathbf{t})}{|K| \mathbf{r}^{\mathbf{4}}}\right] \sum_{i<j}\left(L^{(\mathbf{i})(\mathbf{j})}\right)^{2}\right\} d s^{2}=\sum_{k=1}^{n}\left(d v^{\mathbf{k}}\right)^{2} .
$$

We now define

$$
\exp (-2 \sigma)=\left\{1+\left[\frac{|K| \mathbf{r}^{2}-\mathbf{S}^{2}(\mathbf{r} \sqrt{|\mathbf{K}|} \mathbf{t})}{|K| \mathbf{r}^{4}}\right] \sum_{i<j}\left(L^{(\mathbf{i})(\mathbf{j})}\right)^{2}\right\} .
$$


Placing in (4.13), we obtain

$$
d s^{2}=\exp (2 \sigma) \sum_{k=1}^{n}\left(d v^{\mathbf{k}}\right)^{2} .
$$

We could have all equations in this section on a vielbein basis and the results

would be the same. This will be made at the end of the next section for the general solution.

We rewrite (4.15) as

$$
d s^{2}=\left\{1+\left[\frac{|K| \mathbf{r}^{2}-\mathbf{S}^{2}(\mathbf{r} \sqrt{|\mathbf{K}|} \mathbf{t})}{|K| \mathbf{r}^{4}}\right] \sum_{i<j}\left(\eta_{\mathbf{i j}} L^{(\mathbf{i})(\mathbf{j})}\right)^{2}\right\}^{-1} d v^{\mathbf{l}} d v^{\mathbf{k}} \eta_{\mathbf{l k}},
$$

in which $\eta_{\mathbf{j k}}$ is a generic flat metric.

Through a coordinate transformation, we can put (4.16) in the well-known form

$$
d s^{2}=\left\{1+\frac{K \Omega^{\mathbf{j}} \Omega^{\mathbf{k}} \eta_{\mathbf{j k}}}{4}\right\}^{-2} d \Omega^{\mathbf{j}} d \Omega^{\mathbf{k}} \eta_{\mathbf{j k}} \cdot
$$

But (4.17) is conformal to a flat metric. As (4.16) and (4.17) are equivalent, (4.16) is also conformal to a flat metric.

In the next section, we present some geometric objects in detail, such as normal tensors. This is necessary for the Cartan solution of a general metric.

\section{Normal Tensors}

In this section, Taylor's expansion will be built in the origin of normal coordinates for the metric tensor components. Normal tensors are very important for this. For the covariant derivative, we use the notation (;).

Consider the line element

$$
d s^{2}=G_{\Lambda \Pi} d u^{\Lambda} d u^{\Pi} .
$$

Its expansion has the general form in the origin of a normal coordinate

$$
\begin{array}{r}
d s^{2}=G_{\lambda \pi} d u^{\lambda} d u^{\pi}=G_{\lambda \pi}(0)+\frac{\partial G_{\lambda \pi}}{\partial u^{\mu}} v^{\mu} t \\
+\frac{1}{2} \frac{\partial^{2} G_{\lambda \pi}}{\partial u^{\mu} \partial u^{\nu}} v^{\mu} v^{\nu} t^{2}+\ldots \ldots,
\end{array}
$$


in which the derivatives are calculated at $u^{\pi}=0$.

Some results are found in [6], [7], but these are not generally simple. Our results are simpler because they are more specific.

Consider the covariant derivative of $G_{\lambda \pi}$ in a normal coordinate system.

For a pseudo-Riemannian space, we have

$$
G_{\lambda \pi} ; \mu=0
$$

From (5.3), we obtain

$$
\frac{\partial G_{\lambda \pi}}{\partial u^{\mu}}=C_{\mu \lambda}^{\rho} G_{\rho \pi}+C_{\mu \pi}^{\rho} G_{\lambda \rho}
$$

in which

$$
C_{\mu \lambda}^{\rho}(0)=0
$$

and

$$
\frac{\partial G_{\lambda \pi}}{\partial u^{\mu}}(0)=0
$$

in the origin.

In the limit $u=0$, the partial derivatives of (5.4) supply all derivative terms for the expansion (5.2). Each partial derivative of $C_{\mu \lambda}^{\rho}$, calculated in the origin, is a new tensor. These new tensors are denominated normal tensors. We designate the following representation for these tensors:

$$
D_{\mu \lambda \alpha \beta \ldots \gamma}^{\rho}=\frac{\partial^{n} C_{\mu \lambda}^{\rho}}{\partial u^{\alpha} \partial u^{\beta} \ldots \partial u^{\gamma}}(0) .
$$

We conclude from (5.7) that normal tensors are symmetric in the first pair of inferior indices and have a complete symmetry among other inferior indices. 
It is simple to show that

$$
S\left(D_{\mu \lambda \alpha \beta \ldots \gamma}^{\rho}\right)=0
$$

in which $\mathrm{S}$ designates the sum of different normal tensor components. With (5.4),(5.5), (5.6), (5.7) and (5.8), we can calculate all terms of the expansion (5.2).

Deriving (5.5), calculating the limit and using (5.7), we have

$$
\frac{\partial^{2} G_{\lambda \pi}}{\partial u^{\mu} u^{\nu}}=G_{\lambda \rho} D_{\mu \pi \nu}^{\rho}+G_{\pi \rho} D_{\mu \lambda \nu}^{\rho}
$$

There is more than one way of associating the curvature tensor with normal tensors. Next, we present the simplest way we know.

Let us define the components of the Riemannian curvature tensor in normal coordinates:

$$
R_{\mu \lambda \nu}^{\rho}=\frac{\partial\left(C_{\mu \lambda}^{\rho}\right)}{\partial u^{\nu}}-\frac{\partial\left(C_{\mu \nu}^{\rho}\right)}{\partial u^{\lambda}}+C_{\mu \lambda}^{\sigma} C_{\sigma \nu}^{\rho}-C_{\mu \nu}^{\sigma} C_{\sigma \lambda}^{\rho} .
$$

The limit of (5.10) is

$$
R_{\mu \lambda \nu}^{\rho}=D_{\mu \lambda \nu}^{\rho}-D_{\mu \nu \lambda}^{\rho}
$$

in which we have used (5.5) and (5.7).

Using (5.7), (5.8), (5.11) and the symmetries of the Riemannian curvature tensor, we can show that

$$
D_{\mu \lambda \nu}^{\rho}=\frac{1}{3}\left(R_{\mu \lambda \nu}^{\rho}+R_{\lambda \mu \nu}^{\rho}\right)
$$

Using (5.9) and (5.12), we obtain

$$
\frac{\partial^{2} G_{\alpha \beta}}{\partial u^{\gamma} u^{\delta}} u^{\gamma} u^{\delta}=\frac{2}{3} R_{\alpha \gamma \beta \delta} u^{\gamma} u^{\delta}
$$

Through a similar procedure, but a tedious calculation, we obtain

$$
\frac{\partial^{3} G_{\alpha \beta}}{\partial u^{\mu} u^{\nu} u^{\sigma}} u^{\mu} u^{\nu} u^{\sigma}=R_{\alpha \mu \beta \nu ; \sigma} u^{\mu} u^{\nu} u^{\sigma}
$$


Fourth-order derivatives for metric tensors are easy, but very long. So we will not present them here. We can now conclude Taylor's expansion of the metric tensor. First, we rewrite

$$
\begin{array}{r}
G_{\lambda \pi}=G_{\lambda \pi}(0) \\
+\frac{1}{2} \frac{\partial^{2} G_{\lambda \pi}}{\partial u^{\mu} \partial u^{\nu}} v^{\mu} v^{\nu} t^{2} \\
\frac{1}{6} \frac{\partial^{3} G_{\alpha \beta}}{\partial u^{\mu} u^{\nu} v^{\sigma}} v^{\mu} v^{\nu} v^{\sigma} t^{3}+\ldots
\end{array}
$$

Now we place (5.13) and (5.14) in (5.15), obtaining

$$
\begin{array}{r}
G_{\lambda \pi} d u^{\alpha} d u^{\beta}=G_{\alpha \beta}(0) d u^{\alpha} d u^{\beta}+ \\
+\frac{1}{3}\left[R_{\alpha \gamma \beta \delta} t^{2}+\right. \\
\left.+\frac{1}{2} v^{\sigma} R_{\alpha \mu \beta \nu ; \sigma} t^{3}+\ldots\right] v^{\gamma} v^{\delta} d u^{\alpha} d u^{\beta}, .
\end{array}
$$

Using the symmetries of the curvature tensor, we have the following expansion:

$$
\begin{array}{r}
G_{\lambda \pi} d u^{\alpha} d u^{\beta}=G_{\alpha \beta}(0) d u^{\alpha} d u^{\beta}+ \\
+\frac{1}{12}\left[R_{\alpha \gamma \beta \delta} t^{2}+\right. \\
\left.+\frac{1}{2} v^{\sigma} R_{\alpha \gamma \beta \delta ; \sigma} t^{3}+\ldots\right]\left[v^{\gamma} d u^{\alpha}-v^{\alpha} d u^{\gamma}\right]\left[v^{\beta} d u^{\delta}-v^{\delta} d u^{\beta}\right] .
\end{array}
$$

On the hyper-surface $t=1$, we have $d t=0$ and

$$
\begin{array}{r}
G_{\lambda \pi} d u^{\alpha} d u^{\beta}=G_{\alpha \beta}(0) d v^{\alpha} d v^{\beta}+ \\
+\frac{1}{12}\left[R_{\alpha \gamma \beta \delta}+\right. \\
\left.+\frac{1}{2} v^{\sigma} R_{\alpha \gamma \beta \delta ; \sigma}\right]\left[v^{\gamma} d v^{\alpha}-v^{\alpha} d v^{\gamma}\right]\left[v^{\beta} d v^{\delta}-v^{\delta} d v^{\beta}\right],
\end{array}
$$

which is the same as Cartan's result, although through a different way. It is always possible to place a flat metric in a diagonal form. 
This is the case of a metric at the origin of normal coordinates. In this case, we have

$$
E_{\Lambda}^{(\mathbf{A})}(0)=\delta_{\Lambda}^{(\mathbf{A})}
$$

We now present Taylor's expansion of $E_{\Lambda}^{(\mathbf{A})}$ at the origin of a normal coordinate

$$
\begin{gathered}
E_{\Lambda}^{(\mathbf{A})}(u)=\delta_{\Lambda}^{(\mathbf{A})}+ \\
+\frac{\partial\left(E_{\Lambda}^{(\mathbf{A})}\right)}{\partial\left(u^{\alpha}\right)} d u^{\alpha}+\ldots
\end{gathered}
$$

Multiplying (5.18) by the vielbein components and their inverse, using (5.19) and (5.20), we obtain

$$
\begin{array}{r}
d s^{2}=\eta_{(\mathbf{A})(\mathbf{B})} d z^{(\mathbf{A})} d z^{(\mathbf{B})}+ \\
+\frac{1}{12}\left[R_{(\mathbf{A})(\mathbf{B})(\mathbf{C})(\mathbf{D})}+\right. \\
\left.+\frac{1}{2} z^{(\mathbf{M})} R_{(\mathbf{A})(\mathbf{B})(\mathbf{C})(\mathbf{D}),(\mathbf{M})] .}\right] \\
.\left(z^{(\mathbf{B})} d z^{(\mathbf{A})}-z^{(\mathbf{A})} d z^{(\mathbf{B})}\right)\left(z^{(\mathbf{C})} d z^{(\mathbf{D})}-z^{(\mathbf{D})} d z^{(\mathbf{C})}\right),
\end{array}
$$

in which the calculation was made on the hyper-surface $t=1$ and $d t=0$.

Note that the expansion given by (5.21) is an approximated solution of (2.43). Using a perturbation method, Cartan first solved the equations (2.28) and (2.34) and then placed each solution in (2.43).

From an appropriate coordinate transformation, we can put a metric of an n-dimensional manifold of constant curvature in the form

$$
d s^{2}=\left\{1+\frac{K \Omega^{\alpha} \Omega^{\beta} \eta_{\alpha \beta}}{4}\right\}^{-2} d \Omega^{\rho} d \Omega^{\sigma} \eta_{\varrho \sigma} .
$$

We can build an expression between $d \Omega^{\rho}$ and $d z^{(\mathbf{A})}$, as follows:

$$
d \Omega^{\Lambda}=E_{(\mathbf{A})}^{\Lambda} d z^{(\mathbf{A})} .
$$


Then, placing (5.23) in (3.10), we have

$$
\begin{array}{r}
d s^{2}=\{1 \\
+\frac{1}{2}\left[\frac{1}{2}\left(\epsilon_{\beta} B_{\alpha \beta \gamma \delta}\right)\right. \\
\left.\left.+\eta^{\rho \sigma} A_{\rho \alpha \beta} A_{\sigma \gamma \delta)}\right)\right] . \\
\left.. L^{\alpha \beta} L^{\gamma \delta}\right\}^{-1} \eta_{\alpha \beta} d \Omega^{\alpha} d \Omega^{\beta} .
\end{array}
$$

Because (5.22) and (5.24) are conformal to a flat manifold, there is a conformal transformation between them, with a conformal factor, (exp $2 \psi)$. Then

$$
g_{\alpha \beta}^{\prime}=(\exp 2 \psi) g_{\alpha \beta} .
$$

More specifically,

$$
\begin{array}{r}
\left\{1+\frac{1}{2}\left[\frac{1}{2}\left(\epsilon_{\beta} B_{\alpha \beta \gamma \delta}\right)+\right.\right. \\
\left.\left.\left.+\eta^{\rho \sigma} A_{\rho \alpha \beta} A_{\sigma \gamma \delta)}\right)\right] L^{\alpha \beta} L^{\gamma \delta}\right\}= \\
=(\exp 2 \psi)\left\{1+\frac{K \Omega^{\alpha} \Omega^{\beta} \eta_{\alpha \beta}}{4}\right\}^{2} .
\end{array}
$$

This is an important result with some consequences, as we will see later. If we make the Lie derivative of (5.25) in relation to $\xi$, we obtain

$$
L_{\xi} g^{\prime}=\left(2 \xi(\psi) g+L_{\xi} g\right)(\exp 2 \psi) .
$$

We now consider the condition

$$
2 \xi(\psi) g+L_{\xi} g=0
$$

which implies

$$
L_{\xi} g^{\prime}=0
$$

which is a definition of a Killing vector. The solution to (5.29) is well known, obtaining each of the Killing vectors $\xi$. We conclude that each $\xi$ is a Killing vector in (5.22) and a conformal Killing vector in (5.24). The equations (5.28) and (5.29) show how a Killing vector in (5.22) will be a conformal Killing vector in (5.24). Notice that a pseudo-Riemannian metric can be put in the form (5.24) by a Riemannian normal transformation. In (5.24), we have a conformal Killing vector, which is a Killing vector in (5.22). 


\section{Embedding Manifolds of Constant Curva- ture in Flat Manifolds}

There are many procedures for defining or introducing functions, fields and geometric objects in an n-sphere. From a different point of view, Dirac [8] embedded the De Sitter space in a flat five-dimensional manifold. The author considered functions and fields as residing in a flat five-dimensional manifold and constructed a procedure to project these functions and fields in the De Sitter space. The Dirac procedure implies a need for the quantum momentum and quantum angular momentum postulates. Other authors have used Dirac's ideas or variants of these ideas, as in [9], where an $n+1$ dimensional stereographic projection is used in a way in which the quantum angular momentum remains in the n-sphere. This does not happen in our approach, because the quantum angular momentum resides on an $n+1$ dimensional pseudo-sphere, as we shall see.

In this section, we embed the $\mathrm{n}$-dimensional manifold (5.22) in a flat $\mathrm{n}+1$ dimensional manifold, obtaining the quantum angular momentum of a free particle as the geometric result.

We now consider a manifold (5.22) designated by $\mathrm{S}$ embedded in a flat $\mathrm{n}+1$ dimensional manifold. The following constraint is obeyed [10]:

$$
\eta_{\alpha \beta} x^{\alpha} x^{\beta}=K=\epsilon \frac{1}{R^{2}},
$$

in which $\mathrm{K}$ is the scalar curvature of the n-dimensional manifold (5.22) and $\alpha, \beta=(1,2, \ldots, n+1)$ and $\epsilon=(+1,-1)$.

It is suitable to use a local basis $X_{\beta}=\frac{\partial}{\partial\left(x^{\beta}\right)}$.

We consider a constant vector $\mathbf{C}$ in a flat $\mathrm{n}+1$-dimensional manifold given by

$$
\eta_{\alpha \beta} C^{\alpha} X^{\beta}=\eta^{\alpha \beta} C_{\alpha} X_{\beta}=C,
$$

in which $C^{\alpha}$ are constant and $\mathbf{N}$ is an orthonormal vector to $\mathrm{S}$. We use the symbol $<,>$ for the inner product in the flat $\mathrm{n}+1$-dimensional manifold and $<,>^{\prime}$ for $\mathrm{S}$.

A constant vector $\mathbf{C}$ can be decomposed into two parts, one on $\mathrm{S}$ and the other outside, as follows:

$$
C=\bar{C}+<C, N>N .
$$


From the definition of $\mathbf{N}$ and (6.1), we obtain

$$
N^{\alpha}=\frac{x^{\alpha}}{R}
$$

Let us construct the covariant derivative of $\mathbf{C}$. We have a local basis and a diagonal, unitary metric tensor, so that the Christoffel symbols are null. Thus, the covariant derivative of $\mathbf{C}$ in the $\mathbf{Y}$ direction is given by

$$
\nabla_{Y} C=0 .
$$

It is easy to show that

$$
\nabla_{Y} N=\frac{Y}{R} .
$$

The Lie derivative of the metric tensor in $\mathrm{S}$ is given by [10]:

$$
L_{\bar{U}} g^{\prime}=2 \lambda_{U} g^{\prime},
$$

in which $\mathbf{U}$ is a constant vector in the flat manifold and $\lambda_{U}$ is the characteristic function. For $\mathrm{S}$, the characteristic function is given by

$$
\lambda_{U}=-\frac{1}{R}\langle U, N\rangle .
$$

Placing (6.8) in (6.7), we have

$$
L_{\bar{U}} g^{\prime}=-2 \frac{1}{R}<U, N>g^{\prime} .
$$

In the region of $\mathrm{S}$ in which $\langle U, N>$ is not vanished, $\bar{U}$ is a conformal Killing vector and in the region in which $\langle U, N\rangle$ is vanished, $\bar{U}$ is a Killing vector. We now consider another constant vector $\mathbf{V}$ in the flat space. The Lie derivative of its projection on $\mathrm{S}$ is given by

$$
L_{\bar{V}} g^{\prime}=-2 \frac{1}{R}<V, N>g^{\prime} .
$$

As we consider a local basis and constant vectors $\mathbf{U}$ and $\mathbf{V}$, the commutator is given by

$$
[U, V]=0 .
$$


Then,

$$
L_{[\bar{U}, \bar{V}]} g^{\prime}=-2 \frac{1}{R}<[U, V], N>g^{\prime}=0
$$

Regardless of $\bar{U}$ and $\bar{V}$ being Killing or conformal Killing vectors, their commutator is a Killing object. We will now show that the commutator $[\bar{U}, \bar{V}]$ is proportional to the quantum angular momentum of a particle.

Using (6.3) in the commutator of elements of the basis, we obtain

$$
\begin{array}{r}
{[\bar{U}, \bar{V}]=} \\
=U^{\alpha} V^{\beta}\left[X_{\alpha}-<X_{\alpha}, N>N, X_{\beta}-<X_{\beta}, N>N\right]= \\
=U^{\alpha} V^{\beta}\left[\bar{X}_{\alpha}, \bar{X}_{\beta}\right] .
\end{array}
$$

We now calculate the commutator of elements of the basis by parts.

We have by simple calculation

$$
<X_{\alpha}, N>=\frac{1}{R} \eta_{\alpha \beta} x^{\beta}
$$

Placing (6.14) in (6.13), we obtain

$$
\begin{array}{r}
{\left[\bar{X}_{\alpha}, \bar{X}_{\beta}\right]=} \\
=\left[X_{\alpha}, X_{\beta}\right]-\left[X_{\alpha}, \frac{1}{R} \eta_{\beta \sigma} x^{\sigma} N\right]+\left[X_{\beta}, \frac{1}{R} \eta_{\alpha \sigma} x^{\sigma} N\right]+ \\
+\frac{1}{R^{2}}\left[\eta_{\alpha \sigma} x^{\sigma} N, \eta_{\beta \sigma} x^{\sigma} N\right] .
\end{array}
$$

On a local basis, we have

$$
\begin{gathered}
{\left[X_{\alpha}, X_{\beta}\right]=0,} \\
{\left[\eta_{\alpha \sigma} x^{\sigma} N, \eta_{\beta \sigma} x^{\sigma} N\right]=0 .}
\end{gathered}
$$

Placing (6.16) and (6.17) in (6.15), we obtain

$$
\left[\bar{X}_{\alpha}, \bar{X}_{\beta}\right]=
$$




$$
\begin{array}{r}
=\frac{1}{R^{2}}\left(\eta_{\alpha \sigma} x^{\sigma} \frac{\partial}{\partial\left(x^{\beta}\right)}-\eta_{\beta \sigma} x^{\sigma} \frac{\partial}{\partial\left(x^{\alpha}\right)}\right) \\
=\frac{1}{R^{2}}\left(x_{\alpha} \frac{\partial}{\partial\left(x^{\beta}\right)}-x_{\beta} \frac{\partial}{\partial\left(x^{\alpha}\right)}\right) \\
=-i \frac{1}{\hbar} \frac{1}{R^{2}} L_{\alpha \beta} .
\end{array}
$$

Multiplying $L_{\alpha \beta}$ by a vielbein basis, we obtain

$$
\begin{array}{r}
L_{(\mathbf{A})(\mathbf{B})}= \\
=(i \hbar)\left(R^{2}\right) R_{(\mathbf{A})(\mathbf{B})(\mathbf{C})(\mathbf{D})} x^{(\mathbf{D})} \eta^{(\mathbf{C})(\mathbf{M})} \frac{\partial}{\partial\left(x^{\mathbf{M})}\right.} .
\end{array}
$$

in which

$$
\hat{p}_{(\mathbf{M})}=(i \hbar) \frac{\partial}{\partial\left(x^{\mathbf{M}}\right)}
$$

is the quantum momentum operator of a particle and

$$
\begin{array}{r}
R_{(\mathbf{A})(\mathbf{B})(\mathbf{C})(\mathbf{D})}= \\
=\frac{1}{R^{2}}\left[\eta_{(\mathbf{A})(\mathbf{D})} \eta_{(\mathbf{B})(\mathbf{C})}-\eta_{(\mathbf{A})(\mathbf{C})} \eta_{(\mathbf{B})(\mathbf{D})}\right]
\end{array}
$$

is the curvature of $\mathrm{S}$ on the vielbein basis and $\eta_{(\mathbf{A})(\mathbf{C})}$ is diagonal.

We have used the embedding of an $n$-dimensional manifold $\mathrm{S}$ in a flat $\mathrm{n}+1$ dimensional manifold only to obtain the quantum angular momentum operator of a particle.

We can rewrite (6.19) as follows:

$$
\begin{array}{r}
L_{(\mathbf{A})(\mathbf{B})}= \\
=(i \hbar)\left[\eta_{(\mathbf{A})(\mathbf{D})} \eta_{(\mathbf{B})(\mathbf{C})}-\eta_{(\mathbf{A})(\mathbf{C})} \eta_{(\mathbf{B})(\mathbf{D})}\right] . \\
. x_{(\mathbf{D})} \eta^{(\mathbf{C})(\mathbf{M})} \frac{\partial}{\partial\left(x^{\mathbf{M})}\right.} .
\end{array}
$$


Racah [11] has shown that the Casimir operators of semisimple Lie groups can be constructed from the quantum angular momentum (6.19). Each multiplet of a semisimple Lie group can be uniquely characterized by the eigenvalues of the Casimir operators.

Although we have built the quantum angular momentum from classical geometric considerations, we can write the usual expression for an eigenstate of Casimir operator as follows:

$$
\hat{C}|\ldots>=C| \ldots>\text {. }
$$

Next, we calculate the Lie derivative of the so(p,n-p) algebra.

For the Lie group $\mathrm{SO}(\mathrm{p}, \mathrm{q})$, we choose the signature

$(p, q)=(p, n-p)=(-,-,-, \ldots-,+,+, . .+)$,

with the algebra

$$
\begin{aligned}
{\left[L_{(\mathbf{A})(\mathbf{B})}, L_{(\mathbf{C})(\mathbf{D})}\right]=} & -i\left(\eta_{(\mathbf{A})(\mathbf{C})} L_{(\mathbf{B})(\mathbf{D})}+\eta_{(\mathbf{A})(\mathbf{D})} L_{(\mathbf{C})(\mathbf{B})}\right. \\
& \left.+\eta_{(\mathbf{B})(\mathbf{C})} L_{(\mathbf{D})(\mathbf{A})}+\eta_{(\mathbf{B})(\mathbf{D})} L_{(\mathbf{A})(\mathbf{C})}\right) .
\end{aligned}
$$

Considering the Lie derivative

$$
\begin{array}{r}
\mathbf{L}_{\left[L_{(\mathbf{A})(\mathbf{B})}, L(\mathbf{C})(\mathbf{D})\right]} g^{\prime}= \\
=-R^{4}<\left[\left[X_{(\mathbf{A})}, X_{(\mathbf{B})}\right],\left[X_{(\mathbf{C})}, X_{(\mathbf{D})}\right]\right], N>g^{\prime}=0 .
\end{array}
$$

The vielbein for orthogonal Cartesian coordinates is given by

$$
E_{\Lambda}^{(\mathbf{A})}=\delta_{\Lambda}^{(\mathbf{A})}
$$

We then have

$$
\left[X_{(\mathbf{A})}, X_{(\mathbf{B})}\right]=\left[X_{\alpha}, X_{\beta}\right]=0 .
$$

Notice that $g^{\prime}$ in $\mathrm{S}$ is form-invariant in relation to the Killing vector $\xi[12]$ and the algebra of $\mathrm{SO}(\mathrm{p}, \mathrm{n}-\mathrm{p})$. We conclude that the algebra of $\mathrm{SO}(\mathrm{p}, \mathrm{n}-\mathrm{p})$ is 
a Killing object. The same is true for the algebra of the Lie group $\mathrm{SO}(\mathrm{n})$, in which we could choose the signature $(+,+,+\ldots,+,+)$ for $\mathrm{SO}(\mathrm{n})$.

The constraint (6.1) is invariant for many classical groups. For such groups, it is possible to build operators from the combination of the quantum angular momentum operators, which are Killing objects in relation to $g^{\prime}$. Therefore, the metric is form-invariant in relation to this algebra. Some of these groups are discussed in [12] and Cartan's list of irreducible Riemannian globally symmetric spaces in [5].

Notice that we start from a normal coordinate transformation. In other words, in the region in which the transformation (2.4) is well-behaved, we can build (3.10) and we have (5.22) by a conformal transformation, which was essential to obtaining the quantum angular momentum operator from geometry.

The coordinates $X^{\Pi}$ of the orthonormal Cartesian coordinate frame, which appear in (6.1), reside in the flat $\mathrm{n}+1$-dimensional manifold and describe an n-dimensional pseudo-sphere. A free classical particle, which resides on an n-dimensional pseudo-sphere, obeys the same classical angular momentum expression (3.7), which appears in (3.8)and (3.9), obtained in a different context, and obeys $L^{\lambda \alpha} \subset \mathrm{S}$, with $\alpha, \lambda=(1,2, \ldots, n)$. This is not true for the quantum angular momentum $L_{(\mathbf{A})(\mathbf{B})}$ given by $(6.19)$, because in $R_{(\mathbf{A})(\mathbf{B})(\mathbf{C})(\mathbf{D})}$ we have $(A),(B),(C),(D)=(1,2, \ldots, n, n+1)$. This is not easily or always noticed. We now present a detailed explanation. To avoid a confusing notation, we rewrite (6.1) as follows [13]:

$$
\bar{G}_{\Pi \Lambda} X^{\Pi} X^{\Lambda}=K \eta_{\alpha \beta} x^{\alpha} x^{\beta}+z^{2}=1,
$$

in which $\mathrm{K}$ is the scalar curvature of the n-dimensional manifold (5.22), $\alpha, \beta=(1,2, \ldots, n)$ and $\Lambda, \Pi=(1,2, \ldots, n+1)$.

The line element of the flat $\mathrm{n}+1$-dimensional manifold is given by

$$
d s^{2}=\tilde{G}_{\Pi \Lambda} d X^{\Pi} d X^{\Lambda}=\eta_{\alpha \beta} d x^{\alpha} d x^{\beta}+K^{-1} d z^{2},
$$

After a simple calculation, we have the metric of the n-dimensional pseudosphere

$$
g_{\mu \nu}=\eta_{\mu \nu}+\frac{K}{\left(1-K \eta_{\rho \sigma} x^{\rho} x^{\sigma}\right)} \eta_{\mu \alpha} x^{\alpha} \eta_{\nu \beta} x^{\beta},
$$

in which we have chosen a diagonal metric with each $\eta_{\mu \nu}=\epsilon_{\mu} \delta_{\mu \nu}$ as plus or minus Kronecker's delta function. After a long, but simple consideration of 
(6.29) and (6.30), we put the line element (6.29) in the form

$$
d s^{2}=\frac{d \bar{s}^{2}}{\left.\left(1-K x^{2}\right)\left[1+K \sum_{\alpha>\lambda} \eta_{\lambda \rho} \eta_{\alpha \sigma} L^{\lambda \alpha} L^{\rho \sigma}\right\}\right]}
$$

for which, in $(6.31)$, we have $\alpha=(2,3, \ldots \ldots, n)$,

$$
\begin{gathered}
x^{2}=\eta_{\rho \sigma} x^{\rho} x^{\sigma}, \\
L^{\lambda \alpha}=x^{\lambda} \frac{d x^{\alpha}}{d s}-x^{\alpha} \frac{d x^{\lambda}}{d s},
\end{gathered}
$$

and

$$
d \bar{s}^{2}=\eta_{\rho \sigma} d x^{\rho} d x^{\sigma}
$$

The line element of the n-dimensional pseudo-sphere given by (6.31) is in the conformal form and a particle in free motion described by the classical angular momentum (6.33) resides on this n-dimensional pseudo-sphere. We are going to build Riemann's tensor components for the metric (6.30). Placing (6.30) in Christoffel's symbols, after some calculation, we have

$$
\Gamma_{\mu \nu}^{\eta}=K x^{\eta} g_{\mu \nu}
$$

Now consider Riemann's tensor components:

$$
R_{\mu \sigma \nu}^{\alpha}=\partial_{\nu} \Gamma_{\mu \sigma}^{\alpha}-\partial_{\sigma} \Gamma_{\mu \nu}^{\alpha}+\Gamma_{\mu \sigma}^{\eta} \Gamma_{n \nu}^{\alpha}-\Gamma_{\mu \nu}^{\eta} \Gamma_{\sigma \eta}^{\alpha} .
$$

Placing (6.35) in (6.36), we obtain

$$
R_{\lambda \pi \sigma \rho}=K\left(g_{\lambda \rho} g_{\pi \sigma}-g_{\lambda \sigma} g_{\pi \rho}\right) .
$$

In the origin, $x^{\mu}=0$, Riemann's tensor components are given by

$$
R_{\lambda \pi \sigma \rho}=K\left(\eta_{\lambda \rho} \eta_{\pi \sigma}-\eta_{\lambda \sigma} \eta_{\pi \rho}\right)
$$

Notice that an n-dimensional pseudo-sphere is an isotropic, homogeneous manifold. All points on the n-dimensional pseudo-sphere are equivalent. Through continuous coordinate transformations, we get from (6.38) to (6.37). 
Now consider the quantum angular momentum given by (6.19). Multiplying $R_{(\mathbf{A})(\mathbf{B})(\mathbf{C})(\mathbf{D})}$ by an appropriate vielbein basis $E_{\Lambda}^{(\mathbf{A})} E_{\Pi}^{(\mathbf{B})} E_{\Theta}^{(\mathbf{C})} E_{\Xi}^{(\mathbf{D})}$, and using

$$
G_{\Lambda \Pi}=E_{\Lambda}^{(\mathbf{A})} E_{\Pi}^{(\mathbf{B})} \eta_{(\mathbf{A})(\mathbf{B})},
$$

we have

$$
R_{\Lambda \Pi \Omega \Theta}=K\left(G_{\Lambda \Theta} G_{\Pi \Omega}-G_{\Lambda \Omega} G_{\Pi \Theta}\right),
$$

in which $(\Lambda, \Pi, \Theta, \Omega)=(1,2,3, \ldots, n, n+1)$.

Also consider (6.21) as follows:

$$
\begin{array}{r}
R_{(\mathbf{A})(\mathbf{B})(\mathbf{C})(\mathbf{D})}= \\
=K\left[\eta_{(\mathbf{A})(\mathbf{D})} \eta_{(\mathbf{B})(\mathbf{C})}-\eta_{(\mathbf{A})(\mathbf{C})} \eta_{(\mathbf{B})(\mathbf{D})}\right]
\end{array}
$$

in which $((A),(B),(C),(D))=(1,2,3, \ldots ., n, n+1)$. As all points on the $\mathrm{n}+1$ dimensional pseudo-sphere are geometrically equivalent, we can see (6.41) as (6.40) in the origin of the coordinates frame $X^{\Lambda}=0$ or as the vielbein components at any point on the $\mathrm{n}+1$-dimensional pseudo-sphere. We conclude that a free classical massive particle in an n-dimensional pseudo-sphere has classical motion and classical angular momentum constrained to the n-dimensional pseudo-sphere, but has quantum momentum and quantum angular momentum in an n+1-dimensional pseudo-sphere. An isotropic, homogeneous ndimensional pseudo-sphere has an infinite number of Ricci directions. Thus, from the classical point of view, there is uncertainty in the particle position as well as in relation to the classical angular momentum. It is always possible to go back to the classical original metric, removing the classical particle uncertainty. From the quantum point of view, there is an extra dimension, increasing the particle uncertainty in position, quantum momentum and quantum angular momentum.

From the geometric point of view, some operations with the quantum angular momentum, such as sums and products, suggest the same operations with curvature. We define some procedures in differential geometry by operations with quantum angular momentum. For example, consider the algebra of the group $\mathrm{SO}(\mathrm{p}, \mathrm{n}-\mathrm{p})$ given by $(6.24)$, in which

$$
\begin{aligned}
{\left[L_{(\mathbf{A})(\mathbf{B})}, L_{(\mathbf{C})(\mathbf{D})}\right]=} & -i\left(\eta_{(\mathbf{A})(\mathbf{C})} L_{(\mathbf{B})(\mathbf{D})}+\eta_{(\mathbf{A})(\mathbf{D})} L_{(\mathbf{C})(\mathbf{B})}\right. \\
& \left.+\eta_{(\mathbf{B})(\mathbf{C})} L_{(\mathbf{D})(\mathbf{A})}+\eta_{(\mathbf{B})(\mathbf{D})} L_{(\mathbf{A})(\mathbf{C})}\right) .
\end{aligned}
$$


We can place (6.19) in (6.24), obtaining a representation of the algebra in terms of curvature operators. Placing (6.19) in (6.25), in terms of the curvature operators, we have the form-invariance of the metric tensor $g^{\prime}$ in relation to the algebra so(p,n-p). Any other possible operation among quantum angular momenta defined herein can be placed in terms of curvature. This offers some curious procedures in differential geometry by simple operations with quantum angular momentum, which may not be possible or are very difficult using geometric methods. The association between the quantum angular momentum and differential geometry can be useful in both geometry and physics.

\section{Physical Principles Based on Geometric Properties}

In this section, a new postulate is announced and some physical principles are developed. However, we must first to make some considerations regarding the electromagnetic field on an n-dimensional pseudo-sphere.

Consider the anti-symmetric electromagnetic of second rank tensor $F_{\alpha \sigma}$. In relation to a Killing vector $\xi$, the Lie derivative of $g^{\prime \alpha \beta} F_{\alpha \sigma}=0$ is given by

$$
L_{\xi}\left(g^{\prime \alpha \beta} F_{\alpha \beta}\right)=g^{\prime \alpha \beta} L_{\xi}\left(F_{\alpha \beta}\right)=0 .
$$

or, more explicitly,

$$
L_{\xi}\left(g^{\prime \alpha \beta} F_{\alpha \beta}\right)=g^{\prime \mu \nu}\left[\frac{\partial \xi^{\rho}}{\partial x^{\mu}} F_{\rho \nu}+\frac{\partial \xi^{\rho}}{\partial x^{\mu}} F_{\mu \rho}+\xi^{\lambda} \frac{\partial F_{\mu \nu}}{\partial \xi^{\lambda}}\right]=0 .
$$

Using the symmetry of $g^{\prime \mu \nu}$, we have

$$
L_{\xi}\left(g^{\prime \alpha \beta} F_{\alpha \beta}\right)=\frac{1}{2} \cdot g^{\prime \mu \nu}\left[\xi^{\lambda}\left(\frac{\partial F_{\mu \nu}}{\partial \xi^{\lambda}}+\frac{\partial F_{\nu \mu}}{\partial \xi^{\lambda}}\right)\right]=0,
$$

or

$$
L_{\xi}\left(g^{\prime \alpha \beta} F_{\alpha \beta}\right)=g^{\prime \mu \nu}\left[\xi^{\lambda} \frac{\partial(0)}{\partial \xi^{\lambda}}\right]=0 .
$$


From (7.1) and (7.4), we have

$$
L_{\xi}\left(F_{\alpha \beta}\right)=0
$$

Then, in relation to a Killing vector $\xi, F_{\alpha \beta}$ is maximally form-invariant.

A Killing vector $\xi$ obeys

$$
\xi_{\mu ; \nu}+\xi_{\nu ; \mu}=0
$$

After some considerations, placing (7.6) in (7.5) we have [13]

$$
(n-2) F_{\alpha \beta}=0 \text {, }
$$

which, for $n>2$, implies

$$
F_{\alpha \beta}=0 .
$$

Thus, electric and magnetic fields vanish on an n-dimensional pseudo-sphere. From the usual expressions of electric and magnetic fields, such as functions of scalar and vector potentials, we have

$$
A_{\mu}=0
$$

Consider a density of electrical charges on an n-dimensional pseudo-sphere. As all points of the sphere are geometrically equivalent, a density of electrical charges has the same value at all points. Thus, it is a constant.

From Maxwell's equations, we have

$$
F_{; \rho}^{\rho \sigma}=-J^{\sigma} .
$$

Placing (7.8) in (7.10), we obtain

$$
J^{\sigma}=0
$$

In (5.22), we conclude that the sum of all charges is zero and the sum of all currents is also zero.

Consider, on an n-dimensional pseudo-sphere, a maximally form-invariant second rank tensor $B_{\alpha \sigma}$ as (7.5), with non-defined symmetry,

$$
L_{\xi}\left(B_{\alpha \beta}\right)=0 .
$$


After some considerations, we have [13]

$$
(n-2)\left(B_{\alpha \beta}-B_{\beta \alpha}\right)=0
$$

which, for $n>2$, implies

$$
B_{\alpha \beta}=B_{\beta \alpha}=\text { const. } g_{\beta \alpha} .
$$

The only maximally form-invariant tensor of second rank different from zero is the metric tensor times a constant [13].

To obtain the quantum angular momentum from geometric considerations, we have considered only constant vectors in a flat $\mathrm{n}+1$-dimensional manifold. We now reconsider the qualitative analyses of (6.9) made in Section 6. In the region in which $\langle U, N\rangle$ does not vanish, $\bar{U}$ is a conformal Killing vector and, in the region in which it vanishes, $\bar{U}$ is a Killing vector. In other words, we have Killing and conformal Killing vectors residing on an n-dimensional pseudo-sphere. For our purposes, we need only Killing objects as the quantum angular momentum. Next, we present the following postulate:

\section{In (5.22) Nature always chooses Killing objects.}

Based on this postulate, we build four classical principles, one of which is identified as a classical version of Heisenberg's uncertainty principle and another is identified as a classical version of Bohr's non-radiation postulate. The third principle is not new and is associated to the electrical neutrality of a stable system. The fourth can be interpreted as an equivalence between two descriptions of the motion of the particle: The first as motion due to the presence of forces and the second as a consequence of geometry, as in Einstein's gravitation. For such, we assume only constant vectors in an $n+1$ dimensional flat manifold, in which (5.22) is embedded.

Equations (2.28) and (2.34) tell us that the curvature is null at the points in which $A_{(\mathbf{A})(\mathbf{C})(\mathbf{D})}$ and $B_{(\mathbf{A})(\mathbf{B})(\mathbf{C})(\mathbf{D})}$ are null. In this case, the classical angular momenta are unspecified. We conclude that any free particle in a curved manifold is always in motion, with angular momentum not null, regardless of whether or not we consider a physical theory.

Equation (5.24) tells us that $d s^{2}$ is conformal to a flat manifold and to (5.22). An observer in (5.22) will see the space as being homogeneous and isotropic in the small region in which the transformation (2.4) is well-behaved. Under this condition, Ricci principal directions of space are indeterminate; so that 
the position of the particle in this region is uncertain. In the conjugate points of Jacobi's fields, the transformation (2.4) fails because geodesics cross, mix or come into contact one other. Therefore, close to a conjugate point, we will not have indetermination in the Ricci principal directions and the uncertainty in the particle position disappears. If (5.22) is valid at all points of the space, there will be an indetermination of Ricci principal directions at each point and, consequently, a total uncertainty regarding the position of the particle. This resembles a property of Heisenberg's uncertainty principle and could be seen as a classical version.

The metric (5.22) is form-invariant for a displacement $\xi$, which is a Killing vector. From the postulate above, there are only Killing vectors. In this metric, a physical scalar field is constant or zero and anti-symmetric second rank tensors, such as the electromagnetic tensor, are null. Under these conditions, electromagnetic fields are trivial and there is no radiation. In the neighborhood of the conjugate points, normal coordinate transformations fail and there is no indetermination of the Ricci principal directions. Moreover, the electromagnetic fields are not trivial, being a radiative field. This is similar to Bohr's postulate for radiation and could be seen as a classical version. In the region in which the transformation (2.4) is well-behaved, the metric can be put in the form (5.22) and particles are in free motion without forces. We can consider this a principle, creating an equivalence between two descriptions of motion that are possible through normal transformations. The first description, in local coordinates, is related to the conception of force. The second is related to the conception of motion without force.

We believe that this principle moves in the direction of Einstein's dream, as it points to the possibility of thinking in physics without forces, as in Einstein's gravity.

We notice that the conjugate points of Jacobi's fields may be a consequence of geometric singularities, as in the origin of Schwarzschild's geometry [14], in which the curvature diverges. However, it may be due to the construction of the coordinates, as in the case of a maximally symmetric space, in which the curvature is finite at all points. In the latter case, we have an indetermination of the Ricci principal directions, whereas there is no indetermination in the former.

We recall that, in the region in which there are no conjugate points of Jacobi's fields, it is possible to build a transformation (2.4) between the ordinary metric and (5.24) as well as a conformal transformation between (5.24) and 
(5.22). Because $g^{\prime}$ is form-invariant in the region in which (5.22) is defined, there are no fields or radiation. The quantum angular momentum, which is a Killing object and resides in an $\mathrm{n}+1$-dimensional pseudo-sphere, appears as a geometric consequence of embedding (5.22) in a flat $\mathrm{n}+1$-dimensional manifold. Particles will be in a free motion, but, from the classical point of view, confined on an n-dimensional pseudo-sphere, and, from a quantum point of view, confined on an $\mathrm{n}+1$-dimensional pseudo-sphere. In this context, in which forces do not exist, particle confinement is due to the manifold geometry. This resembles Einstein's geometric vision as well as Heisenberg's uncertainty principle in quantum mechanics.

\section{Local Embedding of Riemannian Manifolds in Flat Manifolds}

In section 3, we presented considerations on the regions in which coordinate transformations are well-defined.

Let us rewrite (3.10)

$$
d s^{2}=\exp (2 \sigma) \eta_{(\mathbf{A})(\mathbf{B})} d z^{(\mathbf{A})} d z^{(\mathbf{B})} .
$$

Defining the transformation of coordinates [15],

$$
y^{(\mathbf{A})}=\exp (\sigma) z^{(\mathbf{A})},
$$

with $(A)=(1,2,3, \ldots, n)$,

$$
y^{n+1}=\exp (\sigma)\left(\eta_{(\mathbf{A})(\mathbf{B})} z^{(\mathbf{A})} z^{(\mathbf{B})}-\frac{1}{4}\right),
$$

and

$$
y^{n+2}=\exp (\sigma)\left(\eta_{(\mathbf{A})(\mathbf{B})} z^{(\mathbf{A})} z^{(\mathbf{B})}+\frac{1}{4}\right) .
$$

It is easy to determine that

$$
\eta_{\mathbf{A B}} y^{\mathbf{A}} y^{\mathbf{B}}=0
$$

in which

$$
\eta_{\mathbf{A B}}=\left(\eta_{(\mathbf{A})(\mathbf{B})}, \eta_{(\mathbf{n}+\mathbf{1}),(\mathbf{n}+\mathbf{1})}, \eta_{(\mathbf{n}+\mathbf{2}),(\mathbf{n}+\mathbf{2})}\right)
$$


with

$$
\eta_{(\mathbf{n}+\mathbf{1}),(\mathbf{n}+\mathbf{1})}=1,
$$

and

$$
\eta_{(\mathbf{n}+\mathbf{2}),(\mathbf{n}+\mathbf{2})=-\mathbf{1}} .
$$

By a simple calculation, we find that the line element is given by

$$
d s^{2}=\exp (2 \sigma) \eta_{(\mathbf{A})(\mathbf{B})} d z^{(\mathbf{A})} d z^{(\mathbf{B})}=\eta_{\mathbf{A B}} d y^{\mathbf{A}} d y^{\mathbf{B}} .
$$

Equation (8.5) is a hyper-cone in the $(\mathrm{n}+2)$-dimensional flat manifold. The metric (8.1) was embedded in the hyper-cone (8.5) of the $(\mathrm{n}+2)$-dimensional flat manifold.

\section{References}

[1] Siqueira A.C.V.V., Conformal Form of Pseudo-Riemannian Metrics by Normal Coordinate Transformations, arXiv:math-ph/ 1006.2868v1 (2010) 1-33.

[2] Siqueira A.C.V.V., A Physical Principle Based on Geometric Properties, arXiv:math-ph/ 1006.4303v2 (2010) 1-25.

[3] Cartan E., Leçôns sur la Geometrie des Espaces De Riemann, GauthierVillars, Paris 1946.

[4] Spivak M., A Comprehensive Introduction to Diferential Geometry, 2, Third Edition, Publish or Perish, Inc., Houston, Texas 1999.

[5] Helgason S., Differential Geometry and Symmetric Spaces, Academic Press, New York and London 1962.

[6] Veblen O. and Thomas T.Y., The Geometry of Paths ,Trans. Am. Math. Society. 25 (1923) 551-602.

[7] Eisenhart L.P., Non Riemannian Geometry, Dover Publications, Inc., Mineola, New York 2005.

[8] Dirac P.A.M., The Electron Wave Equation in De-Sitter Space, Ann. Math. 36,(1935) 657-669. 
[9] Banerjee R., Gauge theories on sphere and Killing vectors, Ann. Phys. 311 (2004) 245-264.

[10] Weber W.C. and Goldberg S.I., Conformal Deformations of Riemannian Manifolds, Queen's Papers in Pure and Applied Mathematics-No.16, Queen's University Kingston, Ontaro, (1969) 77-88.

[11] Greiner W. and Muller B., QUANTUM MECHANICS Symmetries, Second Edition, Springer-Verlag New York Berlin Heidelberg,1994.

[12] R.Gilmore, Lie Groups,Lie Algebras, and Some of Their Applications (Dover Publications,Inc,2002)

[13] Weinberg S., Gravitation and Cosmology: Principles and Applications of the General Theory of Relativity, John Wiley Sons, 1972.

[14] Misner C.W., Thorne K.S., and Wheeler J.A., Gravitation, W. H. Freeman and Company, San Francisco, 1973.

[15] Eisenhart L.P., Riemannian Geometry, Eighth Printing, Princeton University Press, Princeton, New Jersey 1997. 